\title{
Alcohol and drug abuse: integrative approaches
}

\author{
James Lake M.D* \\ Department of Psychiatry and Behavioral Sciences, Stanford University Hospital, Stanford, CA, USA
}

Alcohol and nicotine are the most widely abused substances in all countries resulting in untold personal suffering and enormous economic and social costs. A significant percentage of smokers are also alcoholics and also use illegal drugs. Methamphetamine ('speed'), cocaine and heroin are widely abused in some demographic groups. In recent decades abuse of prescription drugs such as sedative-hypnotics and narcotic pain medications has become a major global health problem. Over-prescribing of sedative-hypnotics and other potentially addictive medications by physicians has resulted in a growing population of individuals who are dependent on sedatives and antianxiety medications. Many psychological, social and biological factors determine the risk of developing a substance abuse problem. One current theory relates self-destructive drug seeking behavior to intense feelings of pleasure associated with particular neurotransmitters and brain circuits stimulated by alcohol and drugs.

Craving may be related to rapid decline in brain levels of dopamine and other neurotransmitters resulting in increased drug-seeking behavior. Rapid changes in mood and thinking often accompany intoxication with alcohol and drugs. Physiological symptoms of acute intoxication or withdrawal include sudden changes in blood pressure, breathing and heart rate. Seizures may occur during intoxication or withdrawal from alcohol or drugs. Withdrawal is a syndrome of intense physical discomfort and emotional distress that occurs when the use of alcohol and other substances is abruptly stopped following prolonged heavy use. Individuals who chronically abuse alcohol or other substances are at increased risk for depressed mood, anxiety, memory problems, dementia and insomnia.

\section{Limitations of conventional treatments of alcohol and drug abuse}

Controlled studies and patient surveys show that most existing pharmacological and psychosocial treatments of alcohol and drug abuse or dependence have only a moderate success rate in helping individuals to discontinue a substance of abuse or maintain abstinence. By the same token, cognitive-behavioral therapy and psychosocial approaches used in relapse prevention programs are not very effective. One year after discontinuing the use of alcohol or any substance of abuse approximately one third of individuals continue to abuse the same substance at the previous level, one third use the same or another substance but in a more controlled way, and roughly one third remain abstinent. Following the one year mark, abstinence rates continue to decline. Naltrexone and other opiate blockers haven proven ineffective in reducing the rate of heroin addiction. Only one third of recovering alcoholics who attend regular Alcoholics Anonymous meetings remain sober for more than one year. Mainstream approaches that are even less effective include aversion therapy, confrontation, educational films and general psychotherapy.

\section{Complementary and alternative treatments of alcohol and drug abuse}

The limited effectiveness of available mainstream treatments of alcohol and drug abuse invites serious consideration of non-medication approaches. Research findings suggest that improved nutrition, certain supplements, herbals and other non-pharmacological therapies are beneficial for reducing craving, diminishing symptoms of withdrawal and reducing relapse risk in alcoholics and narcotics abusers. Most published studies have focused on alcohol abuse. A significant percentage of chronic alcoholics and drug addicts are malnourished and are deficient in thiamin, folate, B-6 and important minerals. Studies suggest that alcoholics who improve their general nutrition maintaining sobriety longer.

Natural supplements used to treat alcohol and drug abuse, reduce craving, decrease withdrawal symptoms or reduce the risk of relapse include vitamins, magnesium and zinc, Chinese and Ayurvedic herbal formulas, amino acids and essential fatty acids. Taking certain amino acids including taurine, and L-tryptophan may reduce alcohol craving or consumption and diminish withdrawal symptoms. One study found that taurine in doses up to $3 \mathrm{gm} /$ day significantly decreased alcohol withdrawal symptoms in hospitalized alcoholics undergoing acute detoxification. While most natural supplements have few or mild adverse effects when a quality brand is used at recommended dosages some may have serious adverse effects when taken at inappropriate high dosages or in combination with prescription medications.

Other approaches that are sometimes beneficial for treating substance abuse, managing withdrawal or reducing the risk of relapse include acupuncture, EEG biofeedback, meditation, yoga and virtual reality exposure therapy. Virtual environments are being used to stimulate alcohol, nicotine or cocaine craving followed by desensitization using cognitive-behavioral techniques.

If you are struggling with alcohol or drug abuse, taking a medication that isn't helping you control drug or alcohol use, experiencing adverse effects, or you simply can't afford to continue taking a prescription medication that is working you will benefit from my book Alcohol and Drug Abuse: The Integrative Mental Health Solution. In the book I provide practical information about a variety of non-medication

Correspondence to: James Lake M.D, Department of Psychiatry and Behavioral Sciences, Stanford University Hospital, Stanford, CA, USA, E-mail: egret4@ sbcglobal.net

Received: October 12, 2016; Accepted: October 29, 2016; Published: November 03,2016 
alternatives that will help you manage symptoms of alcohol or drug abuse such as herbals, vitamins and other natural supplements, whole body approaches, meditation and mind-body practices, and energy therapies.

\section{Alcohol and drug abuse: The integrative mental health solution will help you}

- Understand substance abuse better
- Take inventory of your symptoms

- Learn about non-medication treatments of substance abuse

- Develop a customized treatment plan that is right for you

- Re-evaluate your treatment plan and make changes if your initial plan doesn't work

Copyright: (2016 Lake J. This is an open-access article distributed under the terms of the Creative Commons Attribution License, which permits unrestricted use, distribution, and reproduction in any medium, provided the original author and source are credited. 